\title{
Factors Related to Insomnia in Type 2 Diabetics
}

Ho Chan Kim, M.D.

Kosin University Gospel Hospital, Dept. of Psychiatry

\section{Objectives :}

The aims of this study were to know the frequency and clinical characteristics of insomnia, and related variables to insomnia in patients diagnosed with type 2 diabetes.

\section{Backgrounds :}

Insomnia is common in diabetic patients. We examined which factors affect insomnia in diabetic patients.

\section{Materials and methods :}

For 99 patients with type 2 diabetes, structured interviews were performed. Insomnia was evaluated using the Insomnia Severity Index. The subjects were divided into the group of type 2 diabetics with insomnia $(\mathrm{N}=34)$ and those without insomnia $(\mathrm{N}=65)$ at first, and then statistically analyzed.

\section{Results :}

1) Insomnia could be found in $34.34 \%$ of type 2 diabetics.

2) Type 2 diabetics with insomnia had significantly more single or divorced (respectively $11.8 \%, p<0.05$ ), higher total scores of the K-HDRS $(11.76 \pm 5.52, p<0.001)$, shorter total sleep time $(5.35 \pm 2.00$ hours, $\mathrm{p}<0.001)$, and longer sleep latency $(50.29 \pm 33.80$ minutes, $p<0.001)$.

3) The all item scores of the ISI-K in type 2 diabetics with insomnia were significantly higher than those in type 2 diabetics without insomnia, that is,

total (18.38 \pm 2.69$), \quad$ A1 $(2.97 \pm 0.76), \quad$ A2 $\quad(3.06 \pm 0.69), \quad$ A3

(2.76 \pm 0.61$), B(3.18 \pm 0.72), C(2.09 \pm 0.97), D(2.12 \pm 1.09)$ and $E$ $(2.21 \pm 0.81)$ (respectively $p<0.001)$.

4) Variables associated with insomnia in type 2 diabetics were as following.

Age had significant negative correlation with $A 3$ items of the ISI$K \quad(\beta=-0.241, p<0.05)$. Total scores of the K-HDRS had significant positive correlation, while total sleep time had significant negative correlation with all items of the ISI-K (respectively $\mathrm{p}<0.05$ ). Sleep latency had significant positive correlation with total,, A1, B and E item scores of the ISI-K (respectively $\mathrm{p}<0.05$ ).

Conclusions : Insomnia was found in about $1 / 3$ of type 2 diabetics. According to the presence of insomnia, clinical characteristics including sleep quality as well as quantity seemed to be different. Because depression seemed to be the most important factor influencing on insomnia, clinicians should pay attention to early detection and intervention of depression among type 2 diabetics.

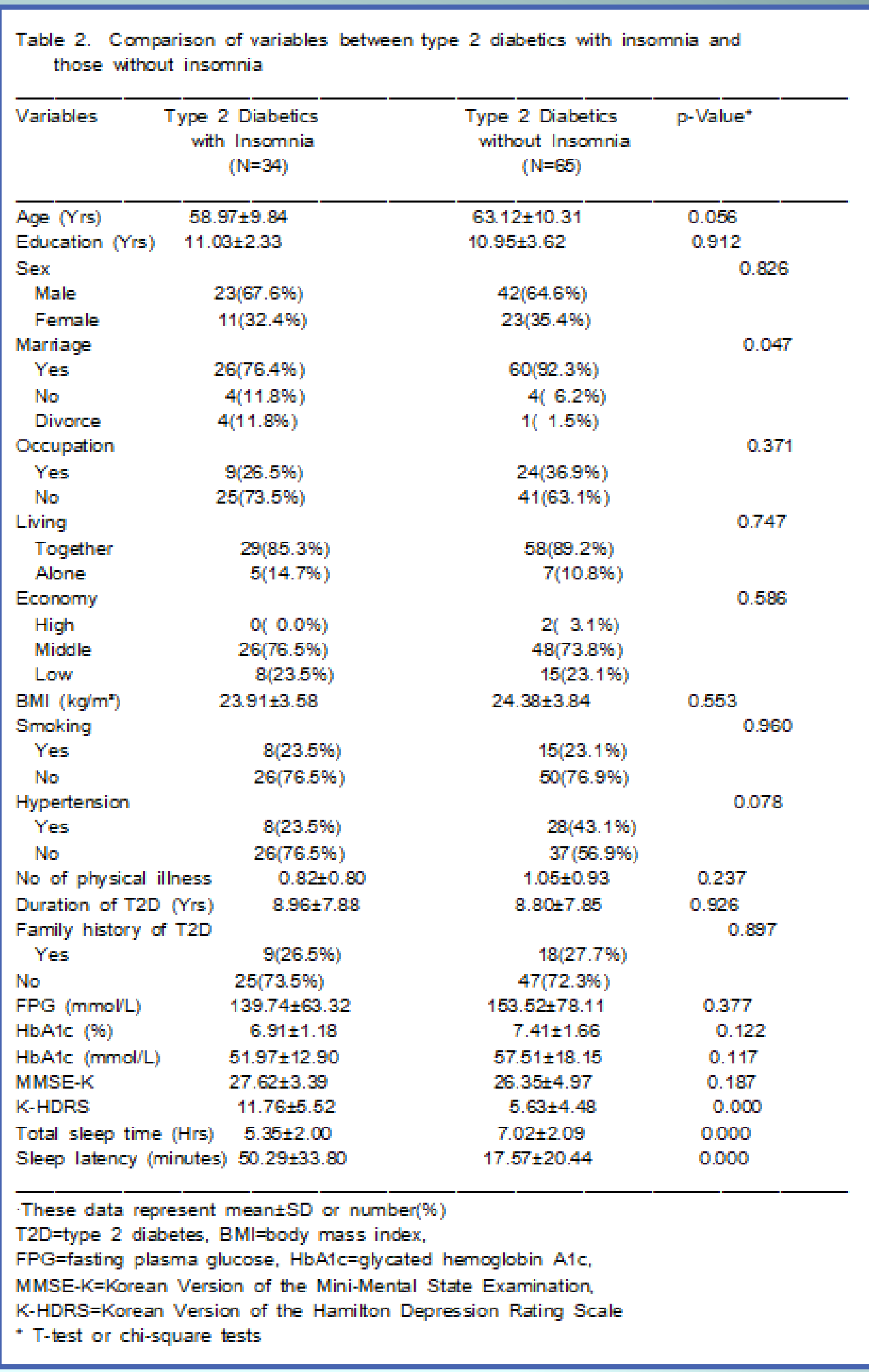

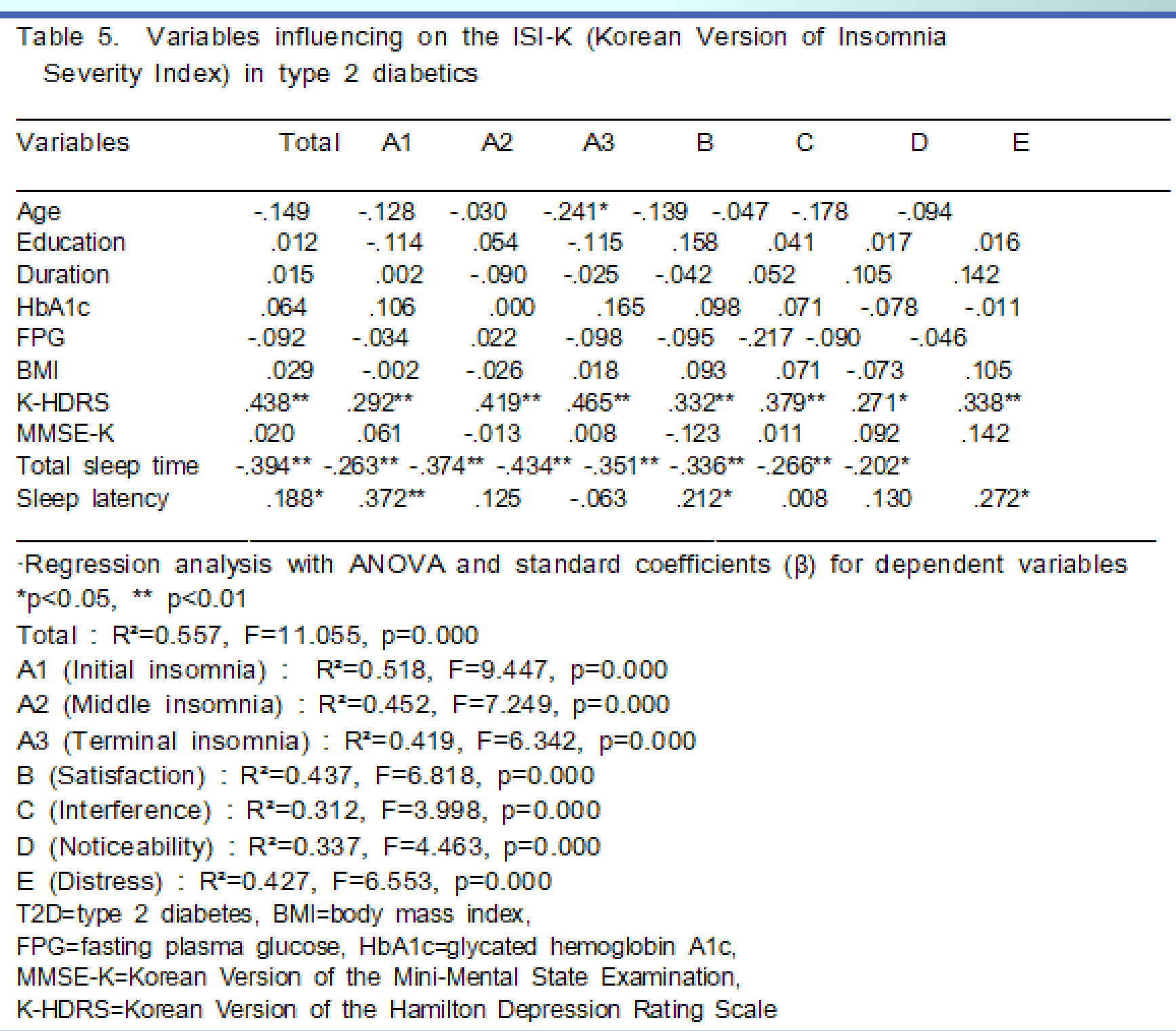

